\title{
Using Interactive Machine Learning to Support Interface Development Through Workshops with Disabled People
}

\author{
Simon Katan \\ Mick Grierson \\ Department of Computing \\ Goldsmiths, University of London \\ s.katan@gold.ac.uk \\ m.grierson@gold.ac.uk
}

Rebecca Fiebrink

r.fiebrink@gold.ac.uk

\begin{abstract}
We have applied interactive machine learning (IML) to the creation and customisation of gesturally controlled musical interfaces in six workshops with people with learning and physical disabilities. Our observations and discussions with participants demonstrate the utility of IML as a tool for participatory design of accessible interfaces. This work has also led to a better understanding of challenges in end-user training of learning models, of how people develop personalised interaction strategies with different types of pre-trained interfaces, and of how properties of control spaces and input devices influence people's customisation strategies and engagement with instruments. This work has also uncovered similarities between the musical goals and practices of disabled people and those of expert musicians.
\end{abstract}

\section{Author Keywords}

Interactive machine learning; accessible interfaces; music.

\section{ACM Classification Keywords}

H5.2. Information interfaces and presentation: User Interfaces.

\section{INTRODUCTION}

Prior work has shown that interactive machine learning (IML) can help people create custom gesturally-controlled systems quickly and without programming, and that these systems can directly encode aspects of embodied practice and "feel right" to use $[2,3]$. This prior work focused primarily on highly skilled computer musicians creating custom gestural interfaces for experimental music. However, very little work has explored the application of IML to user interface design or customisation in other contexts. A better understanding of how IML can be applied by other end users and developers, and of the challenges that can arise, is needed to inform the development of new IML tools and to guide future research.

We present new work investigating the application of IML

Permission to make digital or hard copies of all or part of this work for personal or classroom use is granted without fee provided that copies are not made or distributed for profit or commercial advantage and that copies bear this notice and the full citation on the first page. Copyrights for components of this work owned by others than ACM must be honored. Abstracting with credit is permitted. To copy otherwise, or republish, to post on servers or to redistribute to lists, requires prior specific permission and/or a fee. Request permissions from Permissions@acm.org.

CHI 2015, April 18 - 23, 2015, Seoul, Republic of Korea Copyright 2015 ACM 978-1-4503-3145-6/15/04_..\$15.00 http://dx.doi.org/10.1145/2702123.2702474

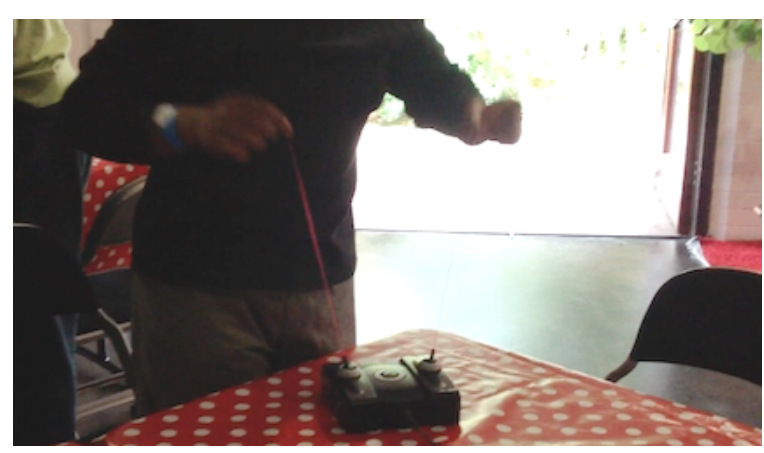

Figure 1: A workshop participant plays a digital instrument built with the Gametrak controller, which senses 3D position of the user's hands using two strings.

to the creation of musical interfaces for people with learning disabilities and physical disabilities. IML is a potentially good match to this domain: disabled people may face insurmountable barriers to playing and becoming proficient on conventional musical instruments, so bespoke computer interfaces can provide a critical creative outlet. However, to our knowledge, IML has not previously been used to create customised interfaces for disabled people, for musical applications or otherwise.

In order to better understand the challenges and benefits of applying IML in this new application space, we partnered with a local community arts centre to conduct a series of workshops in which disabled people interacted with digital musical instruments, built with commercially available and bespoke input devices. IML was used by developers of the instruments and by workshop participants to customise the types of gestures that could be used and the relationships between gesture and sound.

This work presents the first exploration of IML for the development of new interfaces for disabled people. Its primary contributions include insights not only into the challenges and benefits of applying IML to this application space, but also into the ways that properties of the IML process, control space, and input devices impacted on people's customisation of and engagement with gestural interfaces.

\section{RELATED WORK}

Machine learning is potentially useful in the creation of software that must understand or respond to human actions. Instead of writing code, a system designer can provide 
examples of an action (e.g., a body posture sensed by a Kinect) paired with examples of the appropriate computer response (e.g., sending particular control values to a music synthesiser, or sending a "jump" command to a video game avatar). A supervised learning algorithm can then build a model of the relationship between human actions and computer responses, and this trained model can be incorporated into a real-time software system (e.g., changing sound or controlling a game as the user moves).

Initially proposed in the context of computer vision [1], interactive machine learning describes an approach in which a user can iteratively create training examples, examine the resulting trained model, and improve the model by changing the training data and retraining. IML has been investigated by Fiebrink $[2,3]$ in the context of expert musicians building new instruments, by creating training examples from real-time demonstrations of human control movements paired with sounds to be produced by those movements. That work demonstrates that IML can improve interface design by facilitating rapid prototyping, exploration of the design space, and encoding of embodied practices into the interface via the demonstrated examples.

Substantial research has explored approaches for customising interfaces to people with disabilities or impairments, and even early efforts have included datadriven approaches that tailor a device to a user given example measurements of their range of motion [6]. Datadriven methods have also been used to optimise GUIs to individual users [4]. IML has been proposed (but not evaluated) for the end-user customisation of rehabilitation technologies to post-surgery patients [7].

Some high-profile musical projects such as the British Paraorchestra ${ }^{1}$ have engaged engineers to create bespoke instruments for disabled musicians. Most often, though, disabled people who cannot play existing instruments are limited to "accessible" music interfaces such as Soundbeam ${ }^{2}$ or $\mathrm{Skoog}^{3}$. Compared to the instruments that can be built with IML (as used in here), they are still quite limited in terms of customisability and musical breadth.

To our knowledge, this is the first project to apply IML techniques to the creation or customisation of gestural interfaces for people with disabilities. This is also one of the first applications of IML to the creation of gestural control systems with users who are not expert musicians.

\section{METHOD}

\section{IML to Meet Varied Requirements of Disabled Users}

We partnered with a local creative arts centre, Heart n Soul, who work primarily with adults with various combinations of cognitive, physical and sensory impairments. ${ }^{4}$ These

\footnotetext{
${ }^{1}$ http://www.paraorchestra.com/

${ }^{2}$ http://www.soundbeam.co.uk/

${ }^{3}$ http://www.skoogmusic.com/

${ }^{4}$ The centre do not collect information about people's specific disabilities.
}

adults possess a wide diversity of abilities and preferences For example, whilst one person might only be able to execute a narrow range of movement, another might find subtle movements difficult, instead preferring large movements. We sought to discover whether IML could be used to adapt gestural interfaces to people with differing ranges and qualities of movements without loss of functionality. We also sought to explore whether it was feasible and useful for such adaptations to be driven by disabled users themselves within a workshop setting.

\section{A Focus on New Digital Musical Instruments}

Music is an enjoyable and engaging activity for many people. Further, digital instruments (in which a computer generates sound in response to the actions of a person) can incorporate a huge variety of physical actions and real-time control strategies. By building a set of 12 instruments, each using a different pairing of input sensors and musical output, we were able to observe how users' experiences were affected by diverse physical input devices and control strategies (e.g., recognising one of a known set of gestures versus inviting more free-form control; triggering discrete musical events versus offering continuous control over synthesis parameters or effects).

\section{Workshop Structure}

Over two months, we conducted six music-making workshops in collaboration with Heart $n$ Soul. The aims of these workshops were: 1) to create musically engaging experiences for disabled people through the use of digital instruments; 2) to better understand how IML can be used in the development and customisation of such interfaces, and what challenges and benefits may arise; and 3) to inform future research and development work employing IML for interface design.

The workshops included four two-hour sessions with two to four disabled participants, where we introduced people to new instruments developed by our team. The other two workshops were "drop-in" sessions at public events lasting around five hours each, featuring refined versions of our prototypes. These allowed us to observe a wider variety of people (approximately 20 attendees, including disabled people from the arts centre and the wider public).

In each workshop, we placed around a large room two or three of our prototype instruments and a similar number of commercial music interfaces (e.g., Thereminis ${ }^{5}$ and iPad sequencer apps, none of which allowed customisation). Participants could move around the room freely, engaging with instruments or listening to others. Members of our team were present to assist and answer questions, but did not provide formal instructions.

Taking into account the diversity of participants' learning and communication difficulties, we sought to understand on

\footnotetext{
${ }^{5}$ http://www.moogmusic.com/products/etherwave-theremins/theremini
} 
an individual basis how people engaged with each instrument. We observed whether someone spent time actively engaged with an interface, whether they chose to return to the interface on future occasions, the finesse of control demonstrated, and the breadth and quality of musical output. We discussed these observations with participants during the sessions when appropriate and possible. In between workshops, our team discussed our observations and session video logs as we prepared the next set of prototype instruments.

\section{Implementation of Digital Musical Instruments}

Each prototype instrument employed one sensing device: Microsoft Kinect for Xbox (using skeleton tracking to identify limb positions), Leap Motion (in-air hand and finger tracking), video with human face tracking, Gametrak (Figure 1), or one of two squeezable tangibles (stuffed with conductive thread).

To link human actions sensed by these devices to control over sound, we used Wekinator [3], an IML toolkit that allows users to provide training examples of input gestures and matching sounds using real-time demonstration. Our prototype instruments employed the same regression and classification algorithms (neural networks, AdaBoost, knearest neighbour, decision trees, support vector machines) and the same IML workflow as described in previous work $[2,3]$. The outputs from Wekinator's trained models linked people's sensed motions to control over music software created by our team. All instruments allowed sound to be controlled based on the position and/or movement of some part(s) of the player's body (e.g., torso $(x, y, z)$ position or direction of hand motion in front of Kinect). Some instruments offered discrete control (e.g., choosing one of four drum loops) and some continuous control (e.g., sweeping a filter, changing synthesis parameters).

We aimed to design instruments that adhered to the principles of "low threshold" and "high ceiling" [5], where users could make a good sound from the beginning but were rewarded sonically for continuing to gain expertise. We designed instruments to be used to navigate spaces of musical patterns, sound textures, or drum beat sequences, rather than select and trigger individual notes. This allowed people to play in a relatively familiar pop/electronica genre, and to expressively explore a wide variety of sounds, without a fear of playing "wrong" notes. ${ }^{6}$

\section{OUTCOMES}

\section{Challenges of On-the-Fly End-User Customisation}

We initially expected our team to use IML within workshops to modify instruments "on-the-fly," gathering

\footnotetext{
6 Additional information about all instruments is documented at http://doc.gold.ac.uk/ mus02mg/soundlab/
}

training examples directly from participants and training models for each person. We particularly expected this to be helpful for instruments that used classifiers to recognize distinct gestures, since many participants would have trouble reproducing gestures from a pre-determined vocabulary. However, we observed that people frequently had trouble choosing non-ambiguous gestures that could be accurately discriminated by any classifier, had trouble remembering their custom gesture vocabularies, and had trouble performing gestures in a consistent manner. They also had trouble understanding how the training process was used to build the instrument.

\section{How Control Space Properties Impacted Customisation and User Experience}

Nevertheless, we observed that many pre-trained instruments still enabled people to develop effective customisation strategies. These strategies were strongly influenced by the types of control afforded by an instrument, especially whether control was discrete (using gesture recognition to choose among a set of musical events) or continuous (mapping real-time body position or motion properties to a continuous musical parameter space). People engaging with continuous instruments often began by moving in a variety of ways to explore the "sound space" available in an instrument, then gradually settled on a personal gestural vocabulary - a few static positions or types of movement that were relatively easy for them, each resulting in a different sound. People would then play the instrument by switching back and forth among these poses or motions, almost as if the instrument had been trained using a discrete gesture classifier. Unlike the process of training one's own gesture classifier, however, this process allowed people to experiment with a wider variety of gestures, to tailor the set of sounds to their preferences, and to gradually adapt their playing technique without interruptions for model training. People were most successful using this strategy for developing custom control vocabularies when the parts of the continuous musical control space that were accessible using their personal movements offered a large variety in sounds.

Among instruments that recognized discrete gestures, we found that using too many gesture classes (typically, more than 5) produced frustration, as the gesture vocabulary became difficult to remember and classifiers became less accurate. Some of the more engaging discrete instruments used very simple classifiers that left people free to move in ways that were extraneous to the gesture recognition. For example, one popular instrument used centre of mass of a person in front of Kinect to select among four drum samples; many people used their standing position to trivially control the drums while simultaneously dancing expressively to the music.

On the other hand, we observed that instruments with larger discrete control spaces (e.g., selecting among 10-12 percussive samples) could be engaging when they afforded 
use in an exploratory manner, allowing people to discover a variety of sounds and gestures as they moved freely rather than requiring that users memorise and reproduce a specific gesture set. We found that such instruments were easily built by training a regression model and discretising its output, as opposed to providing arbitrarily selected training examples for every discrete "class." Further, the granularity of discretisation could be easily tuned on-the-fly.

\section{IML as a Tool for Developers}

Despite challenges of on-the-fly customisation, our team continued to use IML in our development between workshops due to the speed and flexibility with which new prototypes could be created. Further, IML provided a mechanism by which workshop observations could directly influence prototypes, as developers could train new instruments using example movements similar to those observed. However, when input devices did not expose features that could be used in their raw form to train musically interesting instruments from gestures likely to be used by workshop participants, development dramatically slowed. Possible future work might include the exploration of mechanisms for expediting and automating experimentation with feature normalisation, filtering, and common processing heuristics, with the aim of integrating these with existing IML approaches.

\section{Similarities Between User Groups}

We did not observe clear differences between the ways that disabled people from the community centre and members of the public engaged with our instruments. In fact, our workshop participants' apparent preference for instruments that invited exploration of rich sound spaces using novel (not pre-determined) performer gestures echoes the preferences of expert composers in prior work [3]. This finding reaffirms our supposition that research into the use of gestural interfaces using IML to meet the needs of disabled users is equally useful for the wider community.

\section{CONCLUSION}

Our work demonstrates the utility of employing IML in the design of customised interfaces for disabled people. In exploring this new application area for IML, we draw attention to the following findings that can inform future research related to accessible interfaces and participatory design.

As stated, we observed similarities between the musical goals and practices of people with physical and mental disabilities, and those of expert musicians. This suggests a need for more complex and refined instruments for people with disabilities than those currently commercially available (such as Soundbeam, etc.). This also underscores how existing research on musical expressivity and engagement by musical experts may be relevant to designing instruments for people with disabilities and other user groups.

Our work demonstrates IML's potential value as a design tool, accelerating the design process by allowing the quick translation of participant observations into prototypes. Here, we have highlighted some relevant challenges in using IML in this manner (e.g., participant difficulty in developing memorable gesture vocabularies on the fly) and some ways of addressing those challenges (e.g., using continuous control spaces or larger discrete control spaces that invite exploratory interaction).

Finally, our research shows that the customisation of gestural interfaces can be accomplished not just with custom gesture classifiers tailored to an individual, but by setting up flexible control spaces that allow each user to efficiently explore different motions, simultaneously receiving feedback about how those motions are being interpreted. This allows people to develop new gesture vocabularies that reflect and build on their own abilities, while also accounting for the affordances of the interface, sensors and feature representation. Further, the use of large and flexible control spaces, rather than controls driven by recognition of specific gestures, enables diverse users to experimentally develop bespoke control strategies even while using the same pre-trained interfaces.

\section{ACKNOWLEDGMENTS}

This project was funded by the UK NESTA/AHRC/ACE Digital R\&D programme.

\section{REFERENCES}

1. Fails, J. and D. R. Olsen. 2003. Interactive machine learning. Proc. IUI, 39-45.

2. Fiebrink, R., P. R. Cook, and D. Trueman. 2011. Human model evaluation in interactive supervised learning. Proc. CHI, 147-56.

3. Fiebrink, R., D. Trueman, C. Britt et al. 2010. Toward understanding human-computer interactions in composing the instrument. Int'l Comuter Music Conf.

4. Gajos, K., D.S. Weld and J.O. Wobbrock. 2010. Automatically generating personalized user interfaces with Supple. Artificial Intelligence 174(12-13): 910-50.

5. Myers, B.A., S.E. Hudson and R. Pausch. 2000. Past, present, and future of user interface software tools. $A C M$ Trans. CHI 7(1): 3-28.

6. Pausch, R., and R. D. Williams. 1990. Tailor: Creating custom user interfaces based on gesture. Proc. UIST, 123-34.

7. Xu,Z., R. Fiebrink, and Y. Matsuoka. 2012. Virtual therapist: A Phantom robot-based haptic system for personalised post-surgery finger rehabilitation. Proc. IEEE Robotics \& Biometrics (ROBIO). 